\title{
Utilidad del análisis geográfico en el estudio de las muertes por atropellamiento*
}

\author{
Martha C. Hijar-Medina, Dr. en C.(1)
}

\section{Híjar-Medina MC. \\ Utilidad del análisis geográfico en el estudio de las muertes por atropellamiento. Salud Publica Mex 2000;42:188-193.}

\begin{abstract}
Resumen
Objetivo. Plantear la utilidad de aplicar el análisis geográfico en el estudio de las muertes provocadas por atropellamiento. Material y métodos. Se realizó un estudio transversal, en el año 2000, sobre mortalidad por atropellamiento, de acuerdo con la IX Revisión de la Clasificación Internacional de Enfermedades. Se analizaron certificados de defunción de personas que vivían y fallecieron en la ciudad de México, Distrito Federal, de 1994 a 1997, debido a un atropellamiento. Se calcularon tasas crudas de mortalidad por delegación política y por sexo, asimismo se generaron mapas que contienen las correferencias de las muertes, a distintos grados de agregación, utilizando el programa Map-Info. Resultados D el total de 3687 defunciones por atropellamiento, $71 \%$ correspondieron a personas que vivían en la ciudad de México. Sólo se incluyeron en el proceso de georreferencia 1152 defunciones (43.5\%) que eran las que contaban con información referente al sitio de ocurrencia del atropellamiento. Los resultados, según nivel de agregación, fueron, a nivel delegación política: Milpa Alta, C uajimalpa y Cuauhtémoc, con tasas por arriba de 23/100 000 habitantes; a nivel colonia, 10 presentan concentraciones de nueve y más casos, y a nivel calle, destaca un punto ubicado en LaVenta, en Cuajimalpa, con 25 defunciones. Conclusiones Este tipo de análisis es fundamental desde las per spectivas espacial y epidemiológica al relacionar algunos factores espaciales que coadyuvan en la ocurrencia de atropellamientos. Estos estudios contribuyen al conocimiento y prevención de las muertes por atropellamiento.
\end{abstract}

Palabras clave: accidentes de tránsito/mortalidad; análisis geográfico; México

\author{
Híjar-Medina MC. \\ Geographic analysis \\ of pedestrian run-over injuries. \\ Salud Publica Mex 2000;42:188-193.
}

\begin{abstract}
A bst ract
Objective. The purpose of this paper is to demonstrate the usefulness of geographic analysis to assess the distribution of fatal pedestrian run-over injuries. Material and methods. Cross-sectional study of mortality due to pedestrian run-over injuries was conducted in year 2000. Data were abstracted from death certificates of pedestrians who died due to run-over injuries in Mexico City, during 19941997 (International C lassification of D iseases code E814.7). Crude mortality rates were obtained by political district and gender. Georeferenced mortality data were mapped by different levels of aggregation, using the software program Map-Info. Results.A total of 3687 pedestrian fatalities were reported; $71 \%$ of them were Mexico City residents. 0 nly 1152 deaths (43.5\%) were georeferenced, due to availability of the exact place of the event occurrence. Results by level of aggregation were: by political district level; Milpa Alta, Cuajimalpa and Cuauhtemoc had the highest death rates (23 per 100000 ). At the next level (neighborhoods or colonias), ten colonias had over nine cases; and at the street level, one point at LaVenta in C uajimalpa presented 25 death cases due to pedestrian run-over injuries. Conclusions. This kind of analysis helps us to display the spatial relationship between pedestrian run-over sites and other city landmarks, to advance in the study and prevention of pedestrian run-over deaths.
\end{abstract}

Key words: accidents, traffic/mortality; geographic analysis; Mexico

* Investigación realizada gracias al apoyo del Southern California Injury Prevention Research Center, University of California at Los Angeles (Grant
\#R49 CCR 903622).

(1) Centro de Investigación en Sistemas de Salud, Instituto N acional de Salud Pública, México.

Fecha de recibido: 17 de marzo de 2000 - Fecha de aprobado: 12 de abril de 2000 Solicitud de sobretiros: Martha Híjar Medina. Centro de Investigación en Sistemas de Salud, Instituto N acional de Salud Pública. Avenida Universidad 655, colonia Santa María A huacatitlán, 62508 Cuernavaca, Morelos, México.

Correo electrónico: mhijar@ insp3.insp.mx 
$\mathrm{E}$ n México las lesiones provocadas por accidentes de tráfico ocupan el tercer sitio dentro de las diez principales causas de muerte, lugar que se ha mantenido en el periodo comprendido entre 1989 y 1996. Representan $95 \%$ de las muertes que ocurren debido a accidentes de transporte y son, además, la primer causa de muerte en las edades de entre 1 y 64 años. ${ }^{1}$

En el país, el total de muertes por esta causa, registradas durante 1996, fue de 13 543, lo que significa que durante ese año murieron 37 personas diariamente como consecuencia de lesiones provocadas por este tipo de accidentes; en el ámbito mundial, 77\% de dichas muertes ocurren en zonas urbanas. ${ }^{2}$

En el ámbito urbano existe una mezcla de usuarios expuestos a sufrir un accidente de tráfico: los conductores de vehículos de motor, los motociclistas, los ciclistas y los peatones. En este último caso, queda claro el papel del peatón como el usuario más vulnerable al encontrarse, ante un evento accidental, totalmente desprotegido ante la energía generada por el vehículo contra el cual se impacta. ${ }^{3}$

En la ciudad de México, Distrito Federal, las muertes por atropellamiento son la principal causa dentro del capítulo de accidentes de tráfico. Son, además, una de las principales causas de incapacidad, y la población más afectada se encuentra en edad productiva, pues la media de edad es de 45 años para hombres y 51 para mujeres. * Se notifica un promedio de dos defunciones diarias en el Distrito Federal por esta causa, de las cuales se desconoce cuántas reciben atención pre hospitalaria y hospitalaria, pero se calcula que es del orden de 13 por cada defunción, ${ }^{4}$ lo que significaría que además de las defunciones, 26 atropellados diarios requieren algún tipo de atención médica.

El análisis geográfico mediante la ubicación en mapas de la distribución de casos de enfermedades y su asociación con algunas condiciones ambientales identificadas como riesgosas ha sido utilizado muy frecuentemente en la investigación en salud pública. ${ }^{5-8} \mathrm{En}$ el caso concreto de las lesiones por accidentes de tráfico su utilidad ha sido demostrada en otros estudios, ${ }^{9-11}$ debido a la gran importancia que revisten las características geográficas y ambientales en la ocurrencia de este tipo de lesiones.

Sin embargo, y debido a que en el país no existen artículos publicados donde se haya utilizado esta herramienta para estudiar el problema de los accidentes

\footnotetext{
* Híjar MC, Chu L, Kraus J. Cross national comparison of injury mortality: Los Angeles County, California and Mexico City, Mexico. Enviado a la revista International Journal of Epidemio$\log$.
}

de tráfico, el presente trabajo tiene como objetivo principal plantear la utilidad de aplicar el análisis geográfico en el estudio de las muertes provocadas por atropellamientos en la ciudad de México, Distrito Federal, durante el periodo comprendido entre 1994 y 1997.

\section{Material y métodos}

El presente análisis parte de un diseño transversal, de tipo descriptivo, de mortalidad por atropellamientos cuya fuente de información estuvo constituida por los certificados de defunción correspondientes al periodo de entre 1994 y 1997, de personas que vivían y fallecieron en la ciudad de México, Distrito Federal, y que, de acuerdo con la Clasificación Internacional de Enfermedades ${ }^{12}$ en el capítulo de Causas Externas de Traumatismos y Envenenamientos, la causa de muerte ocurrió por colisión entre un vehículo de motor y un peatón (E 814.7). Este estudio se realizó en el año 2000.

Debido a la utilización indistinta de los términos: ciudad de México, Distrito Federal (D.F.) o Zona Metropolitana de la Ciudad de México (ZMCM), para fines del presente análisis se entenderá por ciudad de México, D.F., solamente a las 16 delegaciones políticas de la ciudad de México y a los 8.2 millones de habitantes registrados en ella.

Las variables consideradas en el análisis fueron: edad, sexo, año en que ocurrió la defunción, lugar de la defunción y delegación política. Es importante señalar que el certificado de defunción contiene el dato de sitio de la defunción pero no el del lugar donde ocurrió el atropellamiento, así que, para poder ubicar esta variable indispensable para el análisis sólo se pudieron incluir aquellos casos que fallecieron en el mismo lugar donde ocurrió el atropellamiento. En los casos donde sí se contaba con esta información se registró el domicilio, colonia y delegación donde sucedió el evento accidental.

Se calcularon las tasas crudas de mortalidad por delegación política con sus respectivos intervalos de confianza al $95 \%$ y globales por sexo para el periodo de estudio (1994-1997); se empleó, como denominador, a las proyecciones intercensales de población, elaboradas por el Consejo Nacional de Población (Conapo).

Para todo el análisis se utilizó como población de referencia a la del Distrito Federal, por lo que al realizar el cálculo de las tasas éstas no se ajustaron por edad, ya que dicha población no difiere estructuralmente ni por las categorías de edad ni por delegación política.

Una vez que se identificaron los casos de muerte por atropellamiento que ocurrieron en el lugar donde el evento sucedió, se procedió a realizar la digitalización manual de cada uno de ellos. Debido a que en 
algunos de los certificados, la información registrada era insuficiente, ya fuera que la dirección no tenía registrado el número de la calle, la colonia o el cruce de las calles, fue necesario tomar las decisiones siguientes: a) en los casos donde sólo se tenía la calle y la colonia, se ubicó sobre la calle y dentro de la colonia; b) en los casos donde sólo se registró la colonia, se digitalizó en el centroide de la colonia; c) cuando las referencias de los cruces de calles no coincidían, se ubicó al menos una de ellas, que correspondiera a la colonia referida, y d) cuando sólo se tenía la colonia pero no era posible ubicarla dentro de la delegación registrada, se eliminó el caso.

La información fue procesada utilizando el programa Map-Info, que es sólo uno de los distintos programas que existen para realizar este tipo de análisis. Una vez que se observó la distribución de los casos y con el fin de plantear las distintas dimensiones del problema, según diversos grados de agregación, se generaron mapas en tres niveles: a) nivel delegación, donde se presentan las tasas de defunción por delegación política; b) nivel colonia, a partir de la distribución de los casos por colonias, se decidió agruparlos de manera que dieran cuenta de distintos gradientes de magnitud, partiendo de las que no presentaron ningún caso, hasta las que presentaron nueve casos o más y así poderlos representar gráficamente; una vez realizado lo anterior se procedió a obtener el nivel más agregado que es el c) nivel calle, éste permitió ubicar los cruces o puntos específicos donde se concentraron el mayor número de muertes, y a partir de los cuales se podría realizar un análisis más profundo de algunos factores del medio ambiente físico relacionados en la ocurrencia de un evento accidental que, como en el presente caso, llega a producir la muerte. Se seleccionaron dos de los puntos más conflictivos y se acudió a observar las condiciones del medio ambiente físico que prevalecía en ellos. Se definió como medio ambiente físico, sólo aquellos aspectos de tipo estructural como: existencia de puentes peatonales, semáforos, señales de tránsito (tipo). Los aspectos relacionados con actitudes y comportamiento de los usuarios del tráfico urbano en estas zonas no se analizan en este artículo.

\section{Resultados}

Durante el periodo analizado de entre 1994 y 1997 ocurrieron un total de 3687 defunciones por atropellamientos en la ciudad de México, de las cuales 2648 $(71 \%)$ correspondieron a personas que vivían en ella. Sólo se incluyeron en el proceso de georreferencia 1152 defunciones (43.5\%) que eran las que contaban con la información acerca del sitio de ocurrencia del atropellamiento.
Con base en el total de casos observados se obtuvo una tasa cruda de mortalidad para la ciudad de México de 7.14/100 000 habitantes (IC 95\% 6.85-7.42). Para el sexo masculino la tasa de fue de 10.6/100 000 (IC 95\% 10.1-11.1), y para el femenino de 4.0/100 000 (IC 95\% 3.66-4.24).

En el análisis por delegación las tasas más altas se observan en Milpa Alta, Cuajimalpa y Cuauhtémoc, con tasas por arriba de 23/100 000 habitantes. El 57\% de las muertes por atropellamiento ocurrieron en cuatro delegaciones, siendo la delegación Cuauhtémoc la que presentó el porcentaje más alto (16.5\%).

La distribución de las defunciones para todo el periodo con base en el sitio de ocurrencia se presenta en la figura 1; cabe destacar que hubo diferencias por delegación entre los que murieron en el sitio de ocurrencia y los que fallecieron en una unidad médica, lo que influyó en los alcances del análisis, ya que Milpa Alta, que es la que presenta la tasa de mortalidad más alta, fue la que presentó el mayor porcentaje de defunciones en unidad médica $(70 \%)$, lo que provocó que sólo $30 \%$ de las muertes ocurridas en la misma se pudieron georreferenciar.

$\mathrm{Al}$ analizar el problema a nivel de colonia (figura 2) se observa que el panorama del problema se va haciendo más específico, ya que los puntos críticos se concentran en 10 colonias en las que destacan, en la

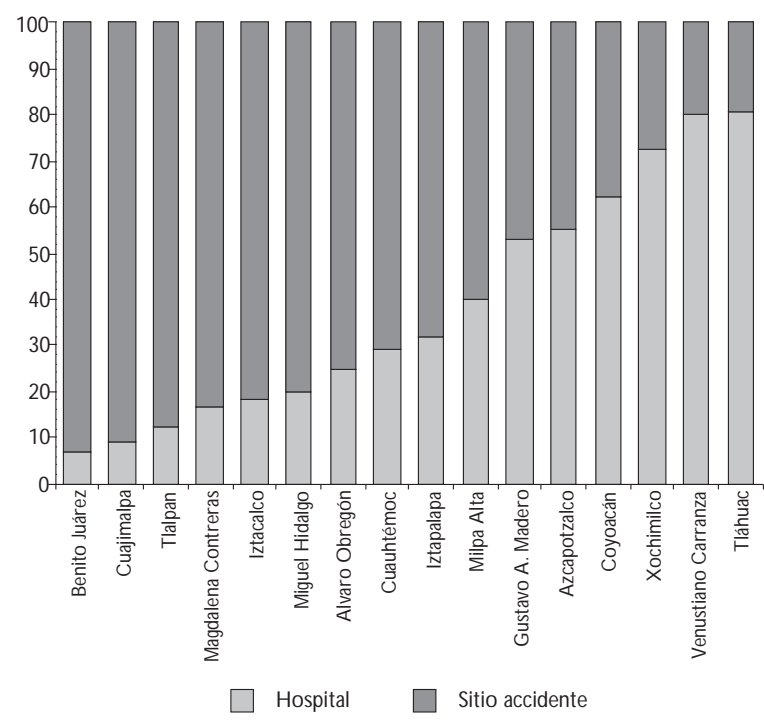

Fuente: certificado de defunción

FigURA 1. Distribución de LAS MUERTES POR ATROPELLA MIENTO, SEGÚN SITIO DE OCURRENCIA DE LA DEFUNCIÓN. CIUdAd de México, D.F., 1994-1997

salud pública de méxico / vol.42, no.3, mayo-junio de 2000 


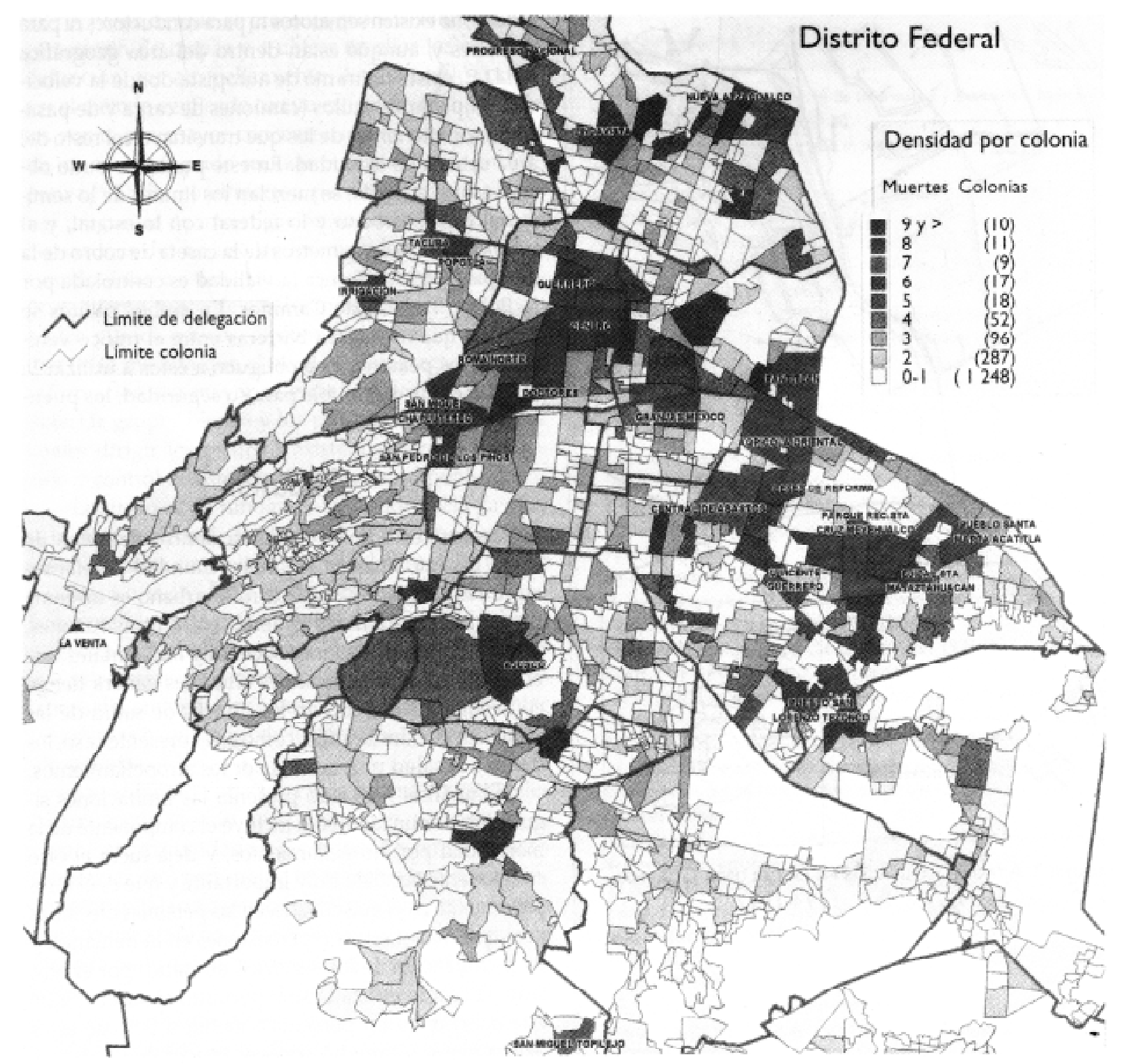

Figura 2. Distribución de las muertes por atropellamiento, según densidad por colonia. Ciudad de México, D.F.,1994-1997

delegación Cuauthémoc, las colonias Centro, Guerrero y Doctores; en la Gustavo A. Madero, Lindavista y Nueva Atzacoalco; en Iztacalco, Agrícola Oriental y Pantitlán; en Iztapalapa, Santa Martha Acatitla y San Lorenzo Tezonco; en Coyoacán, Ajusco, y en Cuajimalpa, La Venta.

$\mathrm{Al}$ analizar al interior de las colonias la agregación a nivel de calle, el problema se fue limitando a seis puntos, de los cuales, con fines de este trabajo, se presentan los resultados de dos de ellos. En el primero, se observa que a lo largo de la avenida Ermita Iztapalapa existen varias concentraciones de eventos. Se seleccionó uno de ellos para la observación posterior. En el segundo destaca, en la delegación Cuajimalpa, un punto ubicado en la zona de La Venta, el cual concentra 25 defunciones (figura 3). En ambos puntos se 


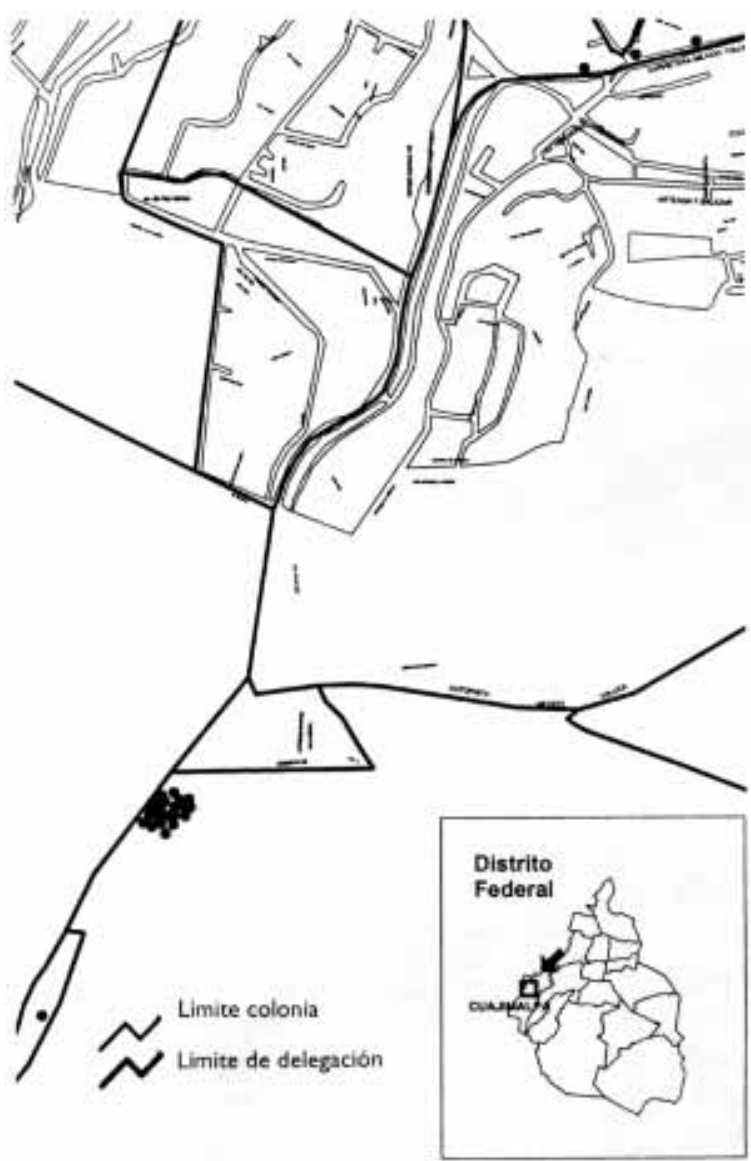

Figura 3. A tropellamientos en la colonia CuajimalPa. Ciudad de México, D.F., 1994-1997

realizó la observación de las características del medio ambiente físico, encontrando lo siguiente: en la avenida Ermita Iztapalapa sí existe puente peatonal de un nivel, pero se encuentra alejado de los puntos de cruce y sólo es utilizado por un bajo número de peatones en relación con el volumen total de ellos. Existen semáforos y señales pero sólo para conductores, el tráfico vehicular está constituido fundamentalmente por vehículos de transporte público (autobuses, microbuses o peseras) y autos particulares.

En el caso de La Venta, en Cuajimalpa, se observó una situación semejante respecto de la señalización, además de dos diferencias fundamentales, a pesar de que también existe puente peatonal, éste tiene cuatro niveles, no existen semáforos ni para conductores ni para peatones $\mathrm{y}$, aunque están dentro del área geográfica del D.F., existe un tramo de autopista donde la velocidad y tipo de vehículos (camiones de carga y de pasajeros) son diferentes de los que transitan en el resto del área urbana de la ciudad. En este punto, se pudo observar que, además, se mezclan los límites de lo semirrural con lo urbano y lo federal con lo estatal, y al estar ubicado a unos metros de la caseta de cobro de la autopista México-Toluca la vialidad es controlada por la Policía Federal de Caminos. En ambos puntos se observó que no existen barreras entre el tráfico vehicular y los peatones que obliguen a éstos a utilizar la única medida disponible para su seguridad, los puentes peatonales.

\section{Discusión}

El presente estudio se enmarca dentro del tema de salud urbana, pues en el ámbito mundial la relación entre calidad de vida y desarrollo urbano es un tema de actualidad e interés general, entre otras razones, porque las modificaciones sociales y ambientales que el crecimiento de las grandes ciudades genera tienen consecuencias directas en los niveles de salud de las poblaciones ${ }^{13}$ destacando, como en el presente caso, los daños a la salud provocados por los atropellamientos.

El análisis realizado presenta las limitaciones siguientes: por un lado, sólo incluye el componente de la mortalidad por atropellamientos, y deja fuera el otro componente también muy importante y que es el atropellamiento en el que no fallecen las personas afectadas pero que tienen serias repercusiones en la demanda y en la utilización de los servicios de salud; por el otro lado, la fuente utilizada sólo permitió conocer dónde está ocurriendo un porcentaje menor a $50 \%$ de estas defunciones, e impidió conocer la variable lugar de ocurrencia del accidente, que en el caso de los accidentes de tráfico, en general, y de los atropellamientos, en particular, es de fundamental importancia. ${ }^{14,15}$ Este hecho implica la necesidad de pugnar por que esta variable sea de registro obligado en el certificado de defunción en todos los casos de accidente de tráfico, en el que además de señalar dónde ocurre la defunción se deberá registrar el lugar donde ocurrió el evento que desencadenó la muerte. Lo anterior, habría que complementarlo con la información referente a los casos no fatales, con base en lo cual se estuviera en la posibilidad de definir las intervenciones más adecuadas de acuerdo con las características locales donde se está produciendo el accidente. 
A pesar de que el presente análisis se limitó a la generación de mapas y a ubicar en ellos los casos de defunción por atropellamiento, éste permitió combinar el método empleado con investigación cualitativa de las zonas con mayor concentración de casos a partir de los cuales se pudo realizar observación no participativa. ${ }^{16}$ Dicha combinación permitirá plantear nuevas preguntas de investigación en torno al análisis de las razones del no uso de las medidas de seguridad cuando éstas existen -puentes peatonales-, del comportamiento, tanto de conductores como de peatones, en los puntos críticos, etcétera. Asimismo, deja claro que en la medida que el nivel de desagregación de la información sea mayor se estará en posibilidades de detectar grupos blanco y los puntos críticos hacia los cuales dirigir los recursos existentes para la prevención y control de este problema de salud pública.

Lo dicho es de fundamental importancia ya que, si bien el análisis de los factores de riesgo de atropellamiento se ha circunscrito principalmente a los planos biológico o individual, se reporta que los países que han experimentado los mayores descensos en la mortalidad por atropellamiento se han distinguido porque sus estrategias de prevención se basan en modificaciones del ambiente inseguro, ${ }^{17,18}$ más que en intentar mejorar las habilidades de los peatones para evitar ser atropellados.

Las diferencias de los casos de muerte por delegación según el sitio de la defunción, ya fuera en el lugar o en una unidad médica, puede estar mostrando problemas de oportunidad en la atención médica, tanto prehospitalaria como hospitalaria, que sería necesario documentar con futuras investigaciones donde se pudiera realizar una medición más precisa de esta variable.

Los resultados de este trabajo también permiten reflexionar sobre los hallazgos de otros autores, donde llama la atención el hecho de que el peatón debe ser incluido como un elemento fundamental en la definición de políticas en el sector transporte, donde da la impresión de que los vehículos y el incremento de vías a construir cobran mayor importancia que los individuos que las utilizan. ${ }^{19-21}$ Esto, además de influir en asegurar un ambiente físico más seguro para el peatón, ${ }^{22-24}$ fomentaría una conducta de respeto al peatón entre los conductores y de responsabilidad sobre autoseguridad entre los propios peatones.

\section{Agradecimientos}

A la licenciada Patricia Ezeta por su participación en la digitalización y elaboración de los mapas.

\section{Referencias}

1. Secretaría de Salud. Dirección de Informática y Evaluación. Mortalidad 1996. México, D.F.: SSA, 1998:69-80.

2. Roberts IG. International trends in pedestrian injury mortality. Arch D is Child 1993; 68(2):190-192.

3. Muhlrad N.Vulnerable road users in urban traffic: Some conclusions of an $O E C D$ expert group. $4^{\text {th }}$ W orld Conference on Injury Prevention and Control (Abstracts).Amsterdam:W orld Health $O$ rganization, 1998:174-175. 4. Secretaría de Salud. Dirección de Informática y Evaluación. A nuario Estadístico de Accidentes y Lesiones. México, D.F.:SSA, 1991.

5. Schwartz T, Callen J, Silva J. A cluster of Hodking's disease in a small community-evidence for environmental factors. Am J Epidemiol 1978;108:19-26.

6 . A bel U, Becker N. G eographic clusters and common patterns in cancer mortality in the Federal Republic of Germany. Arch Environ Health 1987; 42:51-57.

7. Lam N . G eographical patterns of cancer mortality in China. Soc Sci Med 1986;23:241-247.

8. Rothman K. Clustering of disease. Am J Public Health 1987;77:13-15.

9. Waller JA. Health status and motor vehicle crashes. $N$ Engl J Med 1991;391:554-555.

10. Braddock M, Lapidus G, Cromley E, Cromley R, Burke G, Banco L. Using a Geographic Information System to understand child pedestrian injury. Am J Public Health 1994;84(7):1158-1161.

11. Injury Mortality Atlas of the United States 1979-1987. Atlanta (GA): Centers for Disease Control and Prevention, 1991.

12. O rganización Mundial de la Salud/O rganización Panamericana de la Salud.C lasificación Internacional de Enfermedades IX Revisión.W ashington, D.C.:O MS/O PS, 1975:585-694.

13. Castillo M, Reyes S, ed. Problemas emergentes de la Zona Metropolitana de la Ciudad de México. México, D.F.: UN AM, Consejo Mexicano de Ciencias Sociales, 1997.

14.W aller A, Baker S, Szocka A. C hildhood injury deaths: $N$ ational analysis and geographic variations. Am J Public Health 1989;79:310-315.

15. Roberts I, N orton R, Jackson R, D unn R, Hassall I. Effect of environmental factors on risk of injury of child pedestrians by motor vehicles: $A$ case-control study. BMJ 1995;310(6972):91-94.

16. Hammersley M, Atkinson P. Etnografía: el proceso de análisis. Buenos Aires: Paidós Básica, 1994:191-226.

17. Mohan D. Road safety and complexity in less motorised countries:The way ahead. 4th W orld Conference on Injury Prevention and Control (Abstracts). A msterdam:W orld Health 0 rganization, 1998:173.

18. Roberts I. Reducing road traffic. BMJ 1998;316:242-243.

19. C hías L. Las externalidades como problema emergente del Sistema de Transporte Metropolitano. En Castillo M, Reyes S, ed. Problemas emergentes de la Zona Metropolitana de la Ciudad de México. México, D.F.: UN AM, Consejo Mexicano de Ciencias Sociales, 1997:235-259.

20. Avilés B. Medio ambiente y salud. Problemas emergentes de la Zona Metropolitana de la Ciudad de México. México, D.F.: UNAM, Consejo Mexicano de Ciencias Sociales, 1997: 81.

21. Chías L. La geografía humana en México: institucionalización y desarrollo recientes. Geografía del transporte. México, D.F.: UN AM-Fondo de Cultura Económica, 1994:167-179.

22. Söderlund N, Zwi AB. Traffic-related mortality in industrialized and less developed countries. Bull W orld Health 0 rgan 1995;73(2):175-182. 23.W ilson MH, Shock S. Preventing motor vehicle-occupant and pedestrian injuries in children and adolescents. Curr 0 pin Pediatr 1993;5(3): 284-288.

24. Hoxie RE, Rubenstein I.A re older pedestrians allowed enough time to cross intersections safety? J Am Geriatr Soc 1994;42(4):444-450. 